\title{
A blended learning and teaching model to improve bedside undergraduate paediatric clinical training during and beyond the COVID-19 pandemic
}

\author{
S G Lala, ${ }^{1,2}$ MB BCh, MMed, FCPaed, PhD; A Z George, ${ }^{3}$ BSc, BSc Hons, PhD; D Wooldridge, ${ }^{4} \mathrm{MA}$; G Wissing, ${ }^{4} \mathrm{PhD}$; \\ S Naidoo, ${ }^{4}$ BFA; A Giovanelli, ${ }^{4}$ BFA; J King, ${ }^{4}$ BA; M Mabeba, ${ }^{4}$ BFA, PGCE; Z Dangor, ${ }^{1}$ MB BCh, MMed, FCPaed, PhD
}

\author{
${ }^{1}$ Paediatric Education and Research Ladder, Department of Paediatrics and Child Health, Faculty of Health Sciences, University of the Witwatersrand and \\ Chris Hani Baragwanath Academic Hospital, Johannesburg, South Africa \\ ${ }^{2}$ Perinatal HIV Research Unit, Faculty of Health Sciences, University of the Witwatersrand and Chris Hani Baragwanath Academic Hospital, Johannesburg, South Africa \\ ${ }^{3}$ Centre for Health Science Education, Faculty of Health Sciences, University of the Witwatersrand, Johannesburg, South Africa \\ ${ }^{4}$ Centre for Learning, Teaching and Development, University of the Witwatersrand, Johannesburg, South Africa
}

Corresponding author: S G Lala (sanjay.lala@wits.ac.za)

The 2020 COVID-19 pandemic has severely disrupted paediatric undergraduate bedside clinical training. Facing an uncertain future, we need to be able to adapt to the variable effects of the pandemic on bedside training. During severe conditions, no bedside training is possible, while limited bedside training is possible during less severe conditions. We propose a learning and teaching model for undergraduate paediatric clinical training during and beyond the COVID-19 pandemic to facilitate bedside clinical training in order to maximise students' acquisition of clinical competencies.

Afr J Health Professions Educ 2021;13(1):18-22. https://doi.org/10.7196/AJHPE.2021.v13i1.1447

The current COVID-19 pandemic, which is expected to continue far into 2021, has severely disrupted the undergraduate bedside learning and teaching programme in the Department of Paediatrics at the Chris Hani Baragwanath Academic Hospital (CHBAH) in South Africa. CHBAH is the largest academic hospital affiliated with the University of the Witwatersrand, in Johannesburg. Traditional bedside learning ceased when South African (SA) universities were closed at the beginning of the lockdown in March 2020, leading to a mass swing to emergency remote learning (ERL). While online teaching can augment clinical training, it cannot replace bedside clinical skill training. As the lockdown restrictions have eased, students have gradually returned to the hospital in limited numbers to resume clinical training. However, the clinical learning environment is far from ideal, ${ }^{[1,2]}$ and students are not acquiring the expected clinical competencies.

The impact of the pandemic on clinical training at $\mathrm{CHBAH}$ is similar to that experienced elsewhere (Table 1). In addition, in our situation, clinicianeducators are anxious and want to minimise the time spent in high-risk clinical areas. The use of personal protective equipment (PPE), coupled with brief clinical examinations, has severely limited direct interactions with patients. There is also uncertainty about, among other things, the impact of a second wave of the pandemic, when students fully return to the clinical learning environment, and how to deal with students who contract COVID-19 and miss large parts of their clinical rotations. Chiel et al. explicitly stated that the central tenet of medical education [referring to bedside learning and teaching] must be re-examined' and that 'it is hard to ignore that question of whether trainee education is worth pursuing amid a pandemic. ${ }^{[3]}$

The impact of the pandemic on medical education will endure. In our situation, the lack of comprehensive clinical training immediately affects medical students in the final 3 years of study (years $4-6$ of the 6-year MB BCh degree). Assuming a best-case scenario where bedside clinical training 'normalises' in the future, these undergraduate students will need additional undergraduate training. Affected final (6th)-year students will require additional training during their internship year. While acknowledging hardships caused by the pandemic, the clinical competencies of these students and graduates (through no fault of their own) will be substandard. ${ }^{[2]}$ Without additional training, these deficiencies will persist.

The pandemic has affected bedside clinical training in various ways. When severe restrictions (or levels of lockdown) were in place, bedside clinical training ceased completely. Bedside training did not return to normal when restrictions were eased because clinician-educators continued to work at the pandemic coalface and adherence to COVID-19 safety protocols took precedence over bedside training. This consequence meant that students did not fully re-enter the clinical workspace: students took turns to stay at home and attend hospitals and clinics for limited bedside training.

Prior to the COVID-19 pandemic, at our university, medical students learned about basic sciences in their first year, and mainly anatomy and physiology in their second year. The third and fourth years were probably best described as students undertaking 'integrated body system learning' with associated minor clinical skills training. In their last 2 years of training, students undertook discipline-based, 5- to 6-week block rotations with an emphasis on bedside clinical training, with some lecture-based learning. These clinical blocks were offered either in a single year (for some disciplines) or in both clinical years (for others). Final assessments were undertaken at the end of each clinical block and were characterised by theoretical and clinical (mainly objective structured clinical examination (OSCE)) components. The curriculum specified core topics that students should learn during their clinical rotations; blended learning was not 
Table 1. The impact of COVID-19 on undergraduate bedside clinical training (modified after Chiel et al. $)^{[3]}$ and potential solutions

\section{Limited direct interaction with patients:}

- Clinical encounters with inpatients and outpatients are reduced; parents/ caregivers have restricted access to inpatient wards.

- Continuity relationships -where one gets to know a patient - are disrupted.

\section{Reduced colleague/multidisciplinary team interaction:}

- 'Grand ward rounds' - where colleagues and students interact around a patient's bedside - are absent.

- Live interactions at academic meetings (such as journal clubs and case presentations) have ceased.

\section{Suspension of bedside clinical training:}

- Cancelled clinic visits and decreased inpatient volumes, postponed elective admissions or procedures, and dramatic changes in staffing protocols limit the number of training opportunities and the number of students who can be taught in the hospital.

- The move to online learning and teaching platforms raises several problems such as data access and costs, and clinician-educator and readiness.

Insufficient bedside time to develop effective assessment skills:

- Students (who will soon become interns) who spend minimal time in clinical environments cannot perform efficient and tailored physical examinations, and are therefore unable to make accurate clinical assessments.

\section{Improving interactions with patients:}

- Patient encounters need to be focused, brief and effective. Patient interactions should be maintained telephonically or electronically for continuity of care.

- Opportunities need to be identified to facilitate patient, learner and clinician-educator safety in the clinical workspace.

Improving colleague/multidisciplinary team interaction:

- Professional interactions may continue online/electronically.

\section{Resuming bedside clinical training using blended learning:}

- Expert clinician-educators should demonstrate bedside clinical skills in online courses. Specific clinical skills (selected based on their diagnostic relevance and level of difficulty) should initially be demonstrated. Preference should be given to material that promotes self-directed learning. Next, to develop specified clinical competencies, learners should be given directed instruction on how to integrate physical findings to make a diagnosis.

- High data costs need to be addressed at a co-ordinated institutional, government and service provider level.

- Faculty development is required for the production of effective online learning materials.

- Reform existing curricula. ${ }^{[8]}$

Maximising bedside time to develop effective assessment skills:

- New forms of learning materials and online programmes need to be created; for example, blended learning programmes that facilitate the achievement of clinical competence.

- Faculty development focusing on clinician-educators demonstrating fundamental skills at the bedside is needed; these skills should enable interns to diagnose common and/or life-threatening illnesses.

- Clinician-educators should adopt effective learning approaches when bedside training time is limited. This includes the facilitation of pattern recognition by the student instead of the slower traditional Socratic methods. ${ }^{[9]}$

- Assessment tools and examinations (including ward-based assessments) need to be redesigned for blended learning curricula.

- Students need to learn how to triage effectively during a global pandemic and to develop and strengthen health systems during the pandemic. a major feature of the current curriculum and the curriculum was not specifically designed to promote self-directed learning.

The pandemic has forced clinician-educators to re-examine and re-imagine existing training methods, especially for students in their final 2 years of study, which have a strong emphasis on bedside clinical training. The introduction of online learning and teaching platforms (albeit imposed by the swing to emergency remote teaching (ERT) ${ }^{[4]}$ ) has made clinicianeducators aware of blended learning systems that could improve the quality of bedside clinical training in resource-limited settings. The potential advantages that blended learning systems offer are very likely to extend beyond the post-COVID-19 period. How, then, do we move forward?

We propose a blended learning model for continuing the instruction of fifth- and sixth-year undergraduate bedside clinical training during the different stages of the pandemic. Most clinician-educators could face three stages of severity of the pandemic (or variations thereof) when considering if and how bedside training should occur. The first stage describes the period when no bedside training is possible, the second describes a situation where limited bedside training is possible, and the third envisages a return to pre-pandemic training. Considering that the duration of the pandemic is uncertain (as evidenced by the often worse second wave of infections in the USA and Europe) and that our pre-pandemic bedside teaching conditions were far from ideal, ${ }^{[2]}$ we propose that a blended learning programme be used to continue undergraduate bedside training during and beyond the COVID-19 pandemic.

During the development of our clinical training model, we accepted the following conditions, choices and assumptions about clinical training during and beyond the pandemic:

- We should accept that the quality of clinical training (i.e. the acquisition of clinical competencies and reasoning) will be sub-optimal while safety protocols such as physical distancing are required.

- We should not defer difficult choices that lead to a 'toxic combination of inaction and paralysis. ${ }^{[5]}$ 
- We should design adaptable training programmes that can buffer the uncertainties created by the changing levels of pandemic severity on the learning space and environment.

- We need to incorporate innovative, technologically supported methods to enhance blended learning approaches. ${ }^{[6]}$ Clinicianeducators need to work out which clinical skills and competencies are best learnt online and which are best learnt at the bedside.

Our COVID-19 blended learning model (Fig. 1) shows the different clinical training options that are available during different stages of severity of the pandemic and in the post-pandemic period. Four distinct but sequential training options are available during this period: (i) online learning only; (ii) the learning of specific clinical skills at the bedside; (iii) learners receiving directed instruction - at the bedside with clinicianeducators - on how to integrate physical findings to make a diagnosis, and thus acquiring specific and desired clinical competencies; and (iv) the return to full bedside learning. An example of how the model would be used for undergraduate students to acquire clinical competence in the paediatric respiratory examination is presented as an example in Box 1.

\section{Stage 1: Severe pandemic}

As we and other clinician-educators have witnessed, ${ }^{[1,3]}$ bedside clinical training was not possible during the most severe stage of the pandemic. The only available option was online learning, which necessitated an urgent move to what is termed ERL. The term 'ERL' (or sometimes ERT - emergency remote teaching) is distinguished from 'online learning' because of the lack of time for adequate planning and design ${ }^{[6]}$ highlighting an urgent need to develop effective online training materials to facilitate student engagement. These resources will be useful both in the post-COVID-19 era and if we were to return to severe pandemic conditions in the future.

\section{Stage 2: Moderate-severity pandemic conditions}

In this scenario, our current situation (as of November 2020) is that students have partially

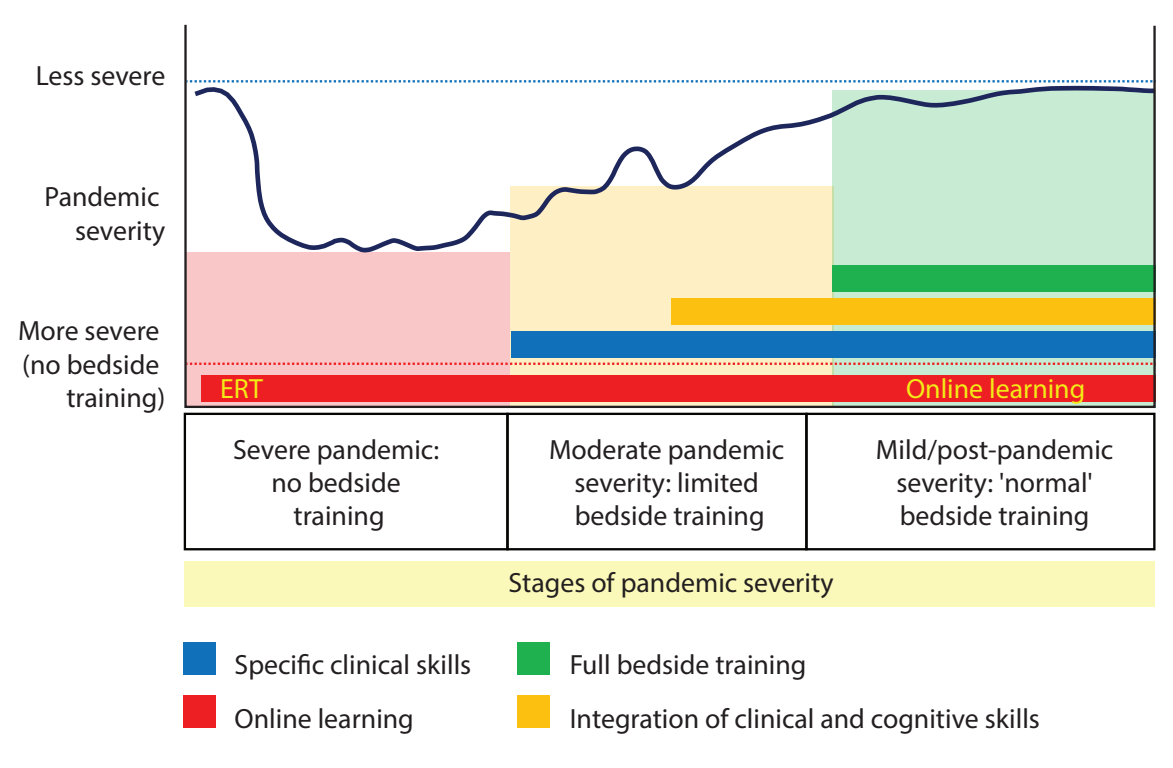

Fig. 1. A blended learning and teaching model for undergraduate paediatric clinical training during and beyond the COVID-19 pandemic. The figure depicts the changing severity (pink, yellow and green blocks) of a hypothetical COVID-19 pandemic (dark blue line) and the variable bedside learning options that are available to students and educators during these times. Online learning (red band shown below the broken red line) is the only option available when students are not allowed in the clinical workspace during severe pandemic conditions. With an easing of pandemic severity, other options become available. If bedside learning is possible but severely limited, specific clinical skills should be learnt (blue band). If bedside training time increases, students can learn to integrate multiple clinical skills to develop specified clinical competencies (yellow band). When learning is unrestricted, full bedside learning becomes possible, but online learning options should be retained to complement bedside clinical training. (ERT = emergency remote teaching.) returned to the clinical workspace. Clinical training consists of ERL and bedside learning. Only a certain number of students are allowed into wards at specified times, to maintain physical distancing. The time spent examining children is brief so that all students have an opportunity to examine children.

Given the limited time at the bedside, online learning is essential to augment clinical training. Inspection skills can be effectively learnt online because well-designed materials can excellently demonstrate physical signs. For example, auscultation skills can be taught with greater effectiveness online, compared with bedside learning, using modern electronic stethoscopes that can record and digitise normal and abnormal lung and heart sounds. The shared listening experience of digital recordings, together with the opportunity for immediate feedback, facilitates deliberate practice. ${ }^{[7]}$ Palpation and percussion techniques can be demonstrated to students by expert clinicians through short videos, and students can practise these skills among themselves with reference to multimedia resources developed for that purpose. The use of online materials to introduce these examination techniques will allow students and educators to focus on the revision of inspection skills and the mastery of specific examination techniques at the bedside.

\section{Stage 3: Mild-severity pandemic or post- pandemic conditions}

As students and clinician-educators have greater access to clinical workspaces, we should determine how best to design online learning and blend it with bedside clinical training. This situation provides an ideal opportunity to review what we teach and how we teach. We need to consider what has worked during the pandemic thus far, and how best to support clinicianeducators in this time of flux.

The educational crisis caused by the pandemic has brought what we teach and how we teach into sharp relief. Although we lamented the lack of complete bedside clinical training during the pandemic, we rarely appreciated its value before the pandemic. ${ }^{[2]}$ There is no alternative to learning bedside clinical skills because there are many limitations of technological diagnostic tests. However, we need to define what clinical skills ranked according to the amount of practice needed to achieve competence - are best taught at the bedside when there is limited time for an examination. 


\section{Box 1. Learning clinical skills to acquire competence in the paediatric respiratory examination}

Online learning: Online programmes serve as the bridge between the textbook and the bedside, and prepare students for bedside practice of clinical skills before they enter the clinical environment. Students will view clinical videos of expert clinicians demonstrating examination techniques and sequences that are relevant to children. Specific techniques that facilitate paediatric examination will be demonstrated to novice students. These techniques include reassuring students that it is acceptable to examine the child on the mother's lap, not to undress the child while ensuring that the examination is thorough and complete, and to avoid 'adult-style' examination techniques that upset children - for example, palpating the trachea, assessing chest wall expansion in infants and young children, and percussing before auscultating the chest.

Programmes should contain clinical recordings (anonymised photographs and videos) of children of various ages with normal breathing patterns and those who demonstrate the abnormal clinical signs associated with respiratory distress (tachypnoea, central cyanosis, alar flaring, head bobbing, opisthotonic posturing, inter- and sub-costal retractions, chest wall indrawing, hyperinflation, etc.). Students can view videos to learn inspection skills; with time, videos can be used to test inspection skills by shortening the viewing time (as an example of purposeful and deliberate practice). The correct percussion techniques, which are often performed incorrectly by doctors, can be demonstrated by expert clinicians; more than one percussion technique can be demonstrated so that students appreciate that clinical skills can be learnt using different approaches. Adaptations for challenging clinical scenarios - for example, the percussion of a young infant's chest - can be demonstrated. Digital stethoscopes can record auscultatory sounds (reflecting both the normal physiological state and those present in various illnesses, including sounds indicating upper airway obstruction such as stridor and steator). In this instance, where the student and teacher are both simultaneously listening to identical auscultatory sounds, online learning is better than bedside learning at providing feedback to the student.

For 5th-year medical students, online programmes can focus on examination techniques, with the demonstration of both normal and abnormal physical signs. For 6th- (or final-) year students, online programmes can focus on clinical diagnosis and management. For both years, material from online programmes can be used to conduct formative and summative assessments. During the level 5 Covid-19 lockdown (i.e. severe restrictions), clinical skill acquisition can only be taught using online programmes.

Clinical skills: Focus on specific clinical skill acquisition at the bedside, using purposeful and deliberate practice. Clinician-educators should prepare for these lessons in advance, focus on clinical skill practice by the student, and refrain from delivering didactic lectures at the bedside. Scripts that facilitate learning of clinical skills and clinical reasoning must be developed.

Example 1: Practice percussion techniques on children of all ages and sizes. Teach the student to examine effectively and timeously - for example, two taps with the plexor finger on the pleximeter finger to determine the percussion note accurately. In the first instance, learn what a normal resonant note sounds like, and then extend one's practice to focus on hyper-resonant and impaired resonance notes. Differentiating dullness from stony dullness will come with practice and experience, and remains difficult in young infants.

Example 2: Teach auscultatory skills in a structured and sequential manner. Novice students are overwhelmed by the plethora of abnormal physical signs present in ill, hospitalised children. They struggle with the interpretation of abnormal signs because they are unsure about physical signs present in the normal physiological state. We favour using a phased approach and check that students are competent at each stage before moving on to the next. For lung auscultation, we begin by assessment of the nature and intensity of breath sounds, followed by detection of any added abnormal sounds. We teach students to differentiate crackles from wheezes first (and teach timing and further specification of these sounds later). We explain that mastery of uncommon and rare sounds (such as bronchial breathing and pleural rubs) comes with practice and experience.

Integration of clinical and cognitive skills: Once competence in specified clinical skills training is achieved, the student is taught to integrate clinical signs to make a diagnosis or formulate a differential diagnosis. Relevant complementary clinical skills are taught - for example, screening for features that suggest right heart failure complicating the respiratory disease.

For 5th-year medical students, the focus is on the acquisition of specific clinical skills until acceptable competence is reached. Final-year students must be able to elicit physical signs, make a diagnosis (or formulate a differential diagnosis), and discuss management principles of common and life-threatening respiratory illnesses. Again, for both years, formative and summative assessments should be conducted during the student's clinical rotation block. During lockdown levels 3 to 4 (i.e. moderate restrictions), specific clinical skill acquisition should be taught to novice medical students entering the clinical workspace, while more senior medical students should be taught to rapidly diagnose common, acute or life-threatening respiratory illnesses in children. Further learning of chronic or non-life-threatening conditions can occur using blended learning programmes.

Full bedside learning: In the final phase, every student (5th or 6th) should have time and opportunity to complete self-directed detailed examinations and further practice and refine clinical skills.

The mastery of clinical skills is not a homogeneous process; some skills are more easily learnt than others. For example, gaining competency in eliciting pedal oedema and detecting scleral jaundice is more straightforward than acquiring mastery in eliciting deep tendon reflexes in children. Other clinical skills are challenging to learn and of limited value in undergraduate training: the detection of moderate ascites using the 'shifting dullness' technique and the accurate characterisation of cardiac murmurs are very difficult to learn during undergraduate paediatric rotations. The value of these clinical skills needs re-appraisal when the use of ultrasonography and echocardiography is required. Therefore, we must define an updated list of essential bedside paediatric clinical skills for undergraduate students. However, the power of clinical examination in making accurate diagnoses must be made clear: students should be strongly encouraged to continuously learn and update their bedside examination techniques throughout their careers. As students become competent in mastering the techniques of specified clinical skills, the interpretation of these clinical signs with deductive reasoning will facilitate clinical diagnosis. To achieve this competency, clinician-educators need to develop teaching and illness scripts that facilitate learning.

Reflecting on the role of ERL during the pandemic, effective online materials must be created, using educational, information technology, media, and content- and clinical-domain experts. Although the financial and temporal investment costs for creating these programmes are significant, the 


\section{Article}

technology can be efficiently scaled up for use by other clinical departments, and online programmes can be co-created and shared with other national and international departments to reduce costs.

Although we have focused on undergraduate students in the present discussion, we need to think about the adverse effects of the pandemic on our current interns too. Current interns have received limited clinical training during their paediatric rotations, and additional training would be required to ensure that these doctors have the desired clinical competency levels if they choose to undergo further training in paediatrics. These doctors can use the same online teaching resources to complement their clinical training.

Our experiences at CHBAH have influenced the development of our model. But we believe that learning and teaching conditions, as well as the effect of the COVID-19 pandemic on clinical training, are similar in many other national and international resource-limited settings. We hope that our proposed blended learning model will benefit educators and students in other settings and that it is used and refined beyond the current pandemic period. At our university, the priority would be to update but shorten our current paediatric undergraduate curriculum using a pedagogic content knowledge framework. ${ }^{[8]}$ Much content can be effectively delivered using online platforms so that the valuable time available for bedside training is used effectively.

Ideally, by mid-2021, we should have created a blended learning programme that facilitates the learning of bedside clinical skills, and developed scripts for learning clinical skills and clinical reasoning. To be clear, our model needs scrutiny and validation and may require modification; but, in these uncertain times, we cannot continue on the current path for much longer and fulfil our mandate to train and graduate competent doctors.
Declaration. None.

Acknowledgements. None.

Author contributions. SGL wrote the first draft. All authors contributed to the development of the blending learning model, read and approved the final draft.

Funding. This work is based, in part, on preparations for creation of a Paediatric Physical Examination Skills (PPES) online instructional programme or massive open online course (MOOC). The PPES MOOC is part of the Open-Access Paediatric Technology Assisted Learning (Open Petal) project, funded by the Discovery Fund (ref. no. 039042). The project is managed through Malamulele Onward NPC.

Conflicts of interest. None.

1. Le Roux C, Stinson K, Dawood F, van Vuuren NJ, Dramowski A. South African medical students' perspectives on COVID-19 and clinical training. Wits J Clin Med 2020;2(3):227-230. https://doi.org/10.18772/26180197.2020. $\mathrm{v} 2 \mathrm{n} 3 \mathrm{a}$

2. George A, Dangor Z, Lala SG. Lamenting the changes in clinical bedside paediatric teaching at Chris Hani Baragwanath Academic Hospital: More resources are needed to train effective doctors. S Afr Med J 2020;110(5):347. https://doi.org/10.7196/SAMJ.2020.v110i5.14678

3. Chiel L, Winthrop Z, Winn AS. The COVID-19 pandemic and pediatric graduate medical education. Pediatrics 2020;146(2):e20201057. https://doi.org/10.1542/peds.2020-1057

4. Affouneh S, Salha S, Khlaif ZN. Designing quality e-learning environments for emergency remote teaching in 4. Affouneh S, Salha S, Khlaif ZN. Designing quality e-learning environm
Coronavirus crisis. Interdiscip J Virtual Learn Med Sci 2020;11(2):1-3.

Coronavirus crisis. Interdiscip J Virtual Learn Med Sci 2020;11(2):1-3.
5. Sneader K, Singhal S. Beyond coronavirus: The path to the next normal. McKinsey and Company. https:// 5. Sneader K, Singhal S. Beyond coronavirus: The path to the next normal. McKinsey and Company. https://
www.mckinsey.com/industries/healthcare-systems-and-services/our-insights/beyond-coronavirus-the-pathto-the-next-normal (accessed 30 September 2020)

6. McCutcheon K, Lohan M, Traynor M, et al. A systematic review evaluating the impact of online or blended learning vs. face-to-face learning of clinical skills in undergraduate nurse education. J Adv Nur 2015;71(2):255-270. https://doi.org/10.1111/jan.12509

. Marcus $\mathrm{CH}$, Newman LR, Winn AS, et al. TEACH and repeat: Deliberate practice for teaching. Clin Teach 2020;17:1-7. https://doi.org/10.1111/tct.13205

8. George A, Dangor Z, Lala SG. South African undergraduate paediatric clinical training: A call to action. S Aft J Child Health 2020;14(1):2-3. https://doi.org/10.7196/SAJCH.2020.v14i1.1755

9. Black CC. Experiential teaching paradigms: Adapting the medical education literature to academic pathology practice. Acad Pathol 2019;6:2374289519892553. https://doi.org/10.1177/2374289519892553

Accepted 11 January 202 